\title{
Optical Fiber Technique for In-Reactor Mechanical Properties Measurement
}

\section{Review of Progress in Quantitative Nondestructive Evaluation}

The INL is a

U.S. Department of Energy

National Laboratory

operated by

Battelle Energy Alliance

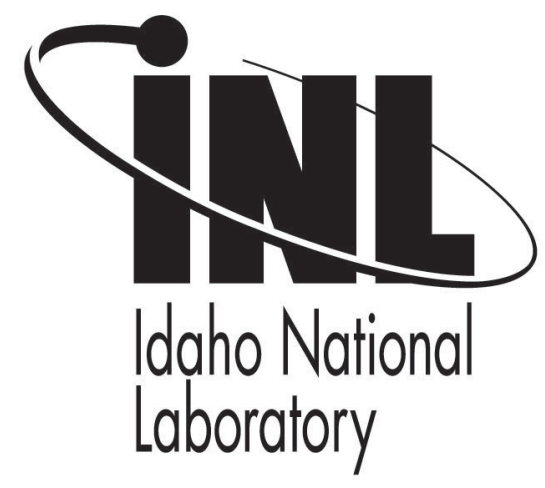

\author{
R. S. Schley \\ D. H. Hurley
}

Z. Hua

July 2012
This is a preprint of a paper intended for publication in a journal or proceedings. Since changes may be made before publication, this preprint should not be cited or reproduced without permission of the author. This document was prepared as an account of work sponsored by an agency of the United States Government. Neither the United States Government nor any agency thereof, or any of their employees, makes any warranty, expressed or implied, or assumes any legal liability or responsibility for any third party's use, or the results of such use, of any information, apparatus, product or process disclosed in this report, or represents that its use by such third party would not infringe privately owned rights. The views expressed in this paper are not necessarily those of the United States Government or the sponsoring agency. 


\title{
OPTICAL FIBER TECHNIQUE FOR IN-REACTOR MECHANICAL PROPERTIES MEASUREMENT
}

\author{
R. S. Schley ${ }^{1}$, D. H. Hurley ${ }^{1}$, and Z. Hua ${ }^{1,2}$ \\ ${ }^{1}$ Dept. of Materials Science, Idaho National Laboratory, Idaho Falls, Idaho 83415, USA \\ ${ }^{2}$ Mechanical and Aerospace Engineering, Utah State University, Logan, Utah 84322, USA
}

\begin{abstract}
In-reactor measurement of material properties is required for a better understanding of radiation effects on materials. We present an optical fiber based technique for measuring changes in elastic properties which involves exciting and measuring flexural vibrations in a thin cantilever beam. By exciting the beam and measuring the resonant frequency, changes in the modulus of elasticity can be monitored. The technique is demonstrated by monitoring the elastic property changes of a beam fabricated from copper, as the copper undergoes recrystallization at elevated temperature.
\end{abstract}

Keywords: Irradiation Testing, Material Properties, Optical Fiber

PACS: $61.80 .-\mathrm{x}$, 46.70.De, 62.40.+i, 81.70.-q, 28.41.Rc, 42.81.Pa

\section{INTRODUCTION}

The development of improved materials for nuclear reactor applications is dependent upon gaining a fundamental understanding of the effects of radiation on the mechanical properties of materials. The real time measurement of mechanical properties in a reactor is however a daunting task for a number of reasons. The environmental conditions in the reactor include very high radiation, with high neutron fluences in both the fast and thermal regimes, as well as high gamma radiation in addition to high temperature and pressure. Material testing positions in a reactor also tend to be small with little space for instrumentation and limited access. Long cable runs are usually required and often must pass through bulkhead fittings at pressure boundaries. Material testing is commonly done using large instruments or fixtures unsuitable for in-reactor testing and most sensors are not able to withstand in-reactor radiation levels. Consequently, the ability to measure changes in material properties during irradiation in a reactor is at present very limited. Indeed, the majority of irradiated material properties measurements are currently made using post irradiation examination (PIE) techniques which provide only before/after information and lack the ability to measure changes in materials as they occur.

Radiation effects materials by displacing atoms which alters the structure and hence the properties of the material. These micro-structural alterations can result in a number of mechanical property changes such as swelling, irradiation hardening, stress corrosion cracking, and imbrittlement among others $[1,2]$. The ability to make in-reactor 
measurements of such radiation effects on material properties has become a priority for a number of programs and will provide valuable new insights into the behavior of nuclear materials exposed to extreme radiation and temperature environments $[3,4]$.

In this work we present an optical fiber based technique for measuring elastic properties in a configuration which is compatible with in-reactor testing. Our approach involves exciting and measuring flexural vibrations in a thin cantilever beam. As a proof of principle, the technique is demonstrated by monitoring the elastic property changes of a copper beam as it undergoes recrystallization at elevated temperature [5].

\section{MEASUREMENT APPROACH}

\section{The Natural Frequency of Vibrating Beams}

The natural frequency of vibrating beams has been used as a means of determining the elastic properties of materials and numerous standards exist detailing such measurements $[6,7,8]$. For a beam fabricated from an isotropic material, the natural frequency depends only on the beam geometry, density and elastic properties. The use of free-free beams is generally specified in these standards in order to minimize boundary condition errors associated with the clamping and pinning of beams in other configurations [9]. However, cantilever beams offer advantages for in reactor measurements in that the beams can be rigidly held in position and alignment with the detection system maintained. By carefully controlling the beam clamping and using an indexing holder as discussed below, the errors associate with the clamped boundary condition can be minimized.

Calculation of the natural frequency for flexural vibrations of a cantilever beam has been broadly discussed in the literature $[10,11,12]$. The simplest formulation is based on Bernoulli-Euler analysis which neglects shear deformation and rotational inertia. Calculation of the natural frequencies based on Bernoulli-Euler formulation can be performed using equation (1) below [13]:

$$
f_{n}=\frac{\left(\beta_{n} l\right)^{2}}{2 \pi l^{2}} \sqrt{\frac{E I}{\rho A}} ; \quad \text { with }\left(\beta_{1} l=1.875, \beta_{2} l=4.694, \beta_{3} l=7.855, \ldots\right)
$$

where $l$ is the beam length, $E$ is the modulus of elasticity, $I$ is the geometrical moment of inertia about the bending axis, $\rho$ is the density, $A$ is the cross sectional area, and $\beta_{n} l$ are solutions to the frequency equation for successive flexural modes with $n$ being the mode number. While less accurate than other theories such as Timoshenko beam theory, this simple formulation

gives accuracy to within a couple percent when $(n / l) *(I / A)^{1 / 2}<0.01$, which is sufficient for the demonstration purposes presented here [14].

\section{Test Capsule Design}

Implementation of this technique required the design of a test capsule with three objectives. First, the capsule needed to be small such that it could be incorporated into an in-reactor test position. Second, the capsule needed to precisely clamp the cantilever beam in position and third the capsule needed to hold optical fibers used for excitation and detection in the proper position. The design of the capsule is shown in Fig. 1. It consists of stainless steel upper and lower halves which could be clamped together using 4-40 socket head cap screws. The sample was sandwiched between the upper and lower halves 


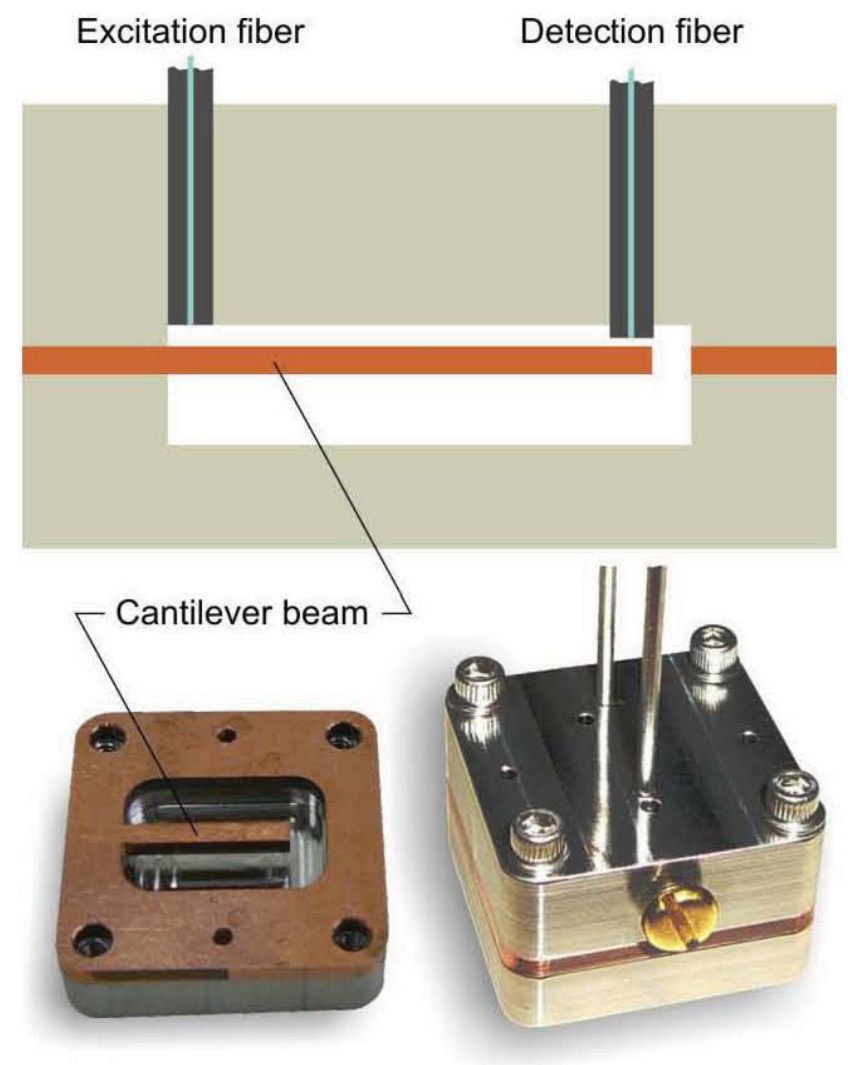

FIGURE 1. Test capsule design. Top - cross section showing the relationship of the cantilever beam to the excitation and detection probes. Bottom - (left) photo of the sample, (right) photo of the assembled capsule.

which contain pockets that allow deflection of the beam. The sample used in this study was fabricated from a thin strip of rolled copper. The copper strip was cut into a $1.125 \mathrm{x}$ 1.125 inch square "frame" with a small cantilever beam machined in the center using electron discharge machining (EDM). Care was taken during the machining to reduce any unnecessary heating of the sample. Holes in the frame allowed clearance for the clamping screws and indexing of the sample with the upper and lower capsule halves using small indexing pins. Indexing of the sample with the capsule halves was important to ensure repeatable positioning and clamping of the cantilever beam. Access holes in the upper half of the capsule allowed 1/16" diameter stainless steel probes containing optical fibers to be positioned over the cantilever beam. The fiber transmitting light for excitation was located above the base of the beam and the detection fiber was located at the tip of the beam.

\section{Detection Technique}

Detection of the beam deflection was accomplished using a fiber optic lever technique [15] which is illustrated in Fig. 2. In this technique light exiting the tip of an optical fiber is reflected from a sample and coupled back into the fiber where it propagates back toward the source. The light exits the fiber tip in divergent cone whose angle of incidence is determined by the numerical aperture of the optical fiber. The intensity of the light returning to the core of the optical fiber is then dependent on the distance between the fiber tip and the sample. Thus, the fluctuation of the tip of the cantilever beam causes an intensity modulation of the light returning back to the detector. Since we are interested only in the frequency of vibration, the absolute intensity of the light is unimportant as is an absolute calibration of the deflection. 


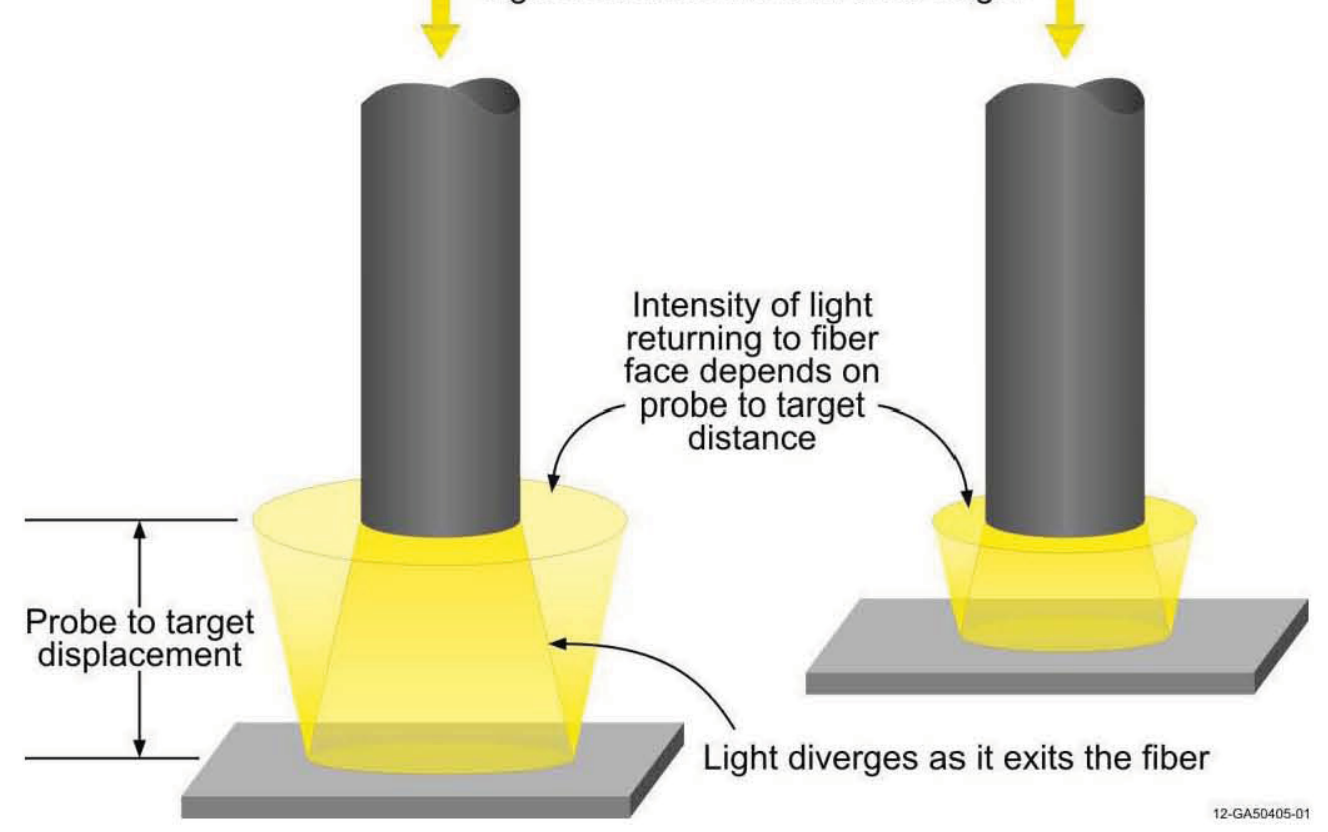

FIGURE 2. Fiber optic lever detection technique showing change in light intensity coupled back into the fiber based on distance to the target

The advantages of this detection technique lie in its simplicity and low cost - it requires only a broadband light source, a fiber splitter and a photoreceiver. The sensitivity of this technique is in the sub micrometer range which is less sensitive than an interferometer, but is less complex and requires no stabilization or expensive optics. This detection method is independence of absolute light intensity which minimizes the effects of radiation induced attenuation in the optical fiber on the measurement, making it a good candidate for in-reactor measurements.

\section{Experiment Configuration}

The experiment layout is shown in Fig. 3. A chopped laser was used to excite the cantilever beam and the vibration of the beam was detected using the technique described above. A network analyzer (HP 3589A) was used to generate a swept frequency signal which was converted to a TTL signal using a Pulse Research Lab model PRL-350TTL. The TTL signal served as the modulation signal for an acousto-optic modulator (Gooch \& Housego 23080-1) which chopped a 532nm laser beam (JDSU M142H-532-500). The chopped laser beam was coupled into a $200 \mu \mathrm{m}$ core optical fiber and delivered to the base of the cantilever beam in the test capsule exciting the cantilever through thermoelastic expansion. The average output power of the excitation laser at the probe tip was about 175 $\mathrm{mW}$. An Ocean Optics HL-2000-FHSA halogen light source was used for detection. This broadband light was coupled into a $200 \mu \mathrm{m}$ core, $0.22 \mathrm{NA}$ optical fiber. The detection light passed through a 50/50 fiber optic splitter and was delivered to the probe tip in the sample capsule. Detection light reflected from the cantilever beam tip propagated back to the 50/50 splitter where half of the light was directed to a photoreceiver (New Focus 2033) whose output signal was returned to the network analyzer for phase locked detection. Probe tips for both generation and detection beams consisted of a gold coated optical fiber 


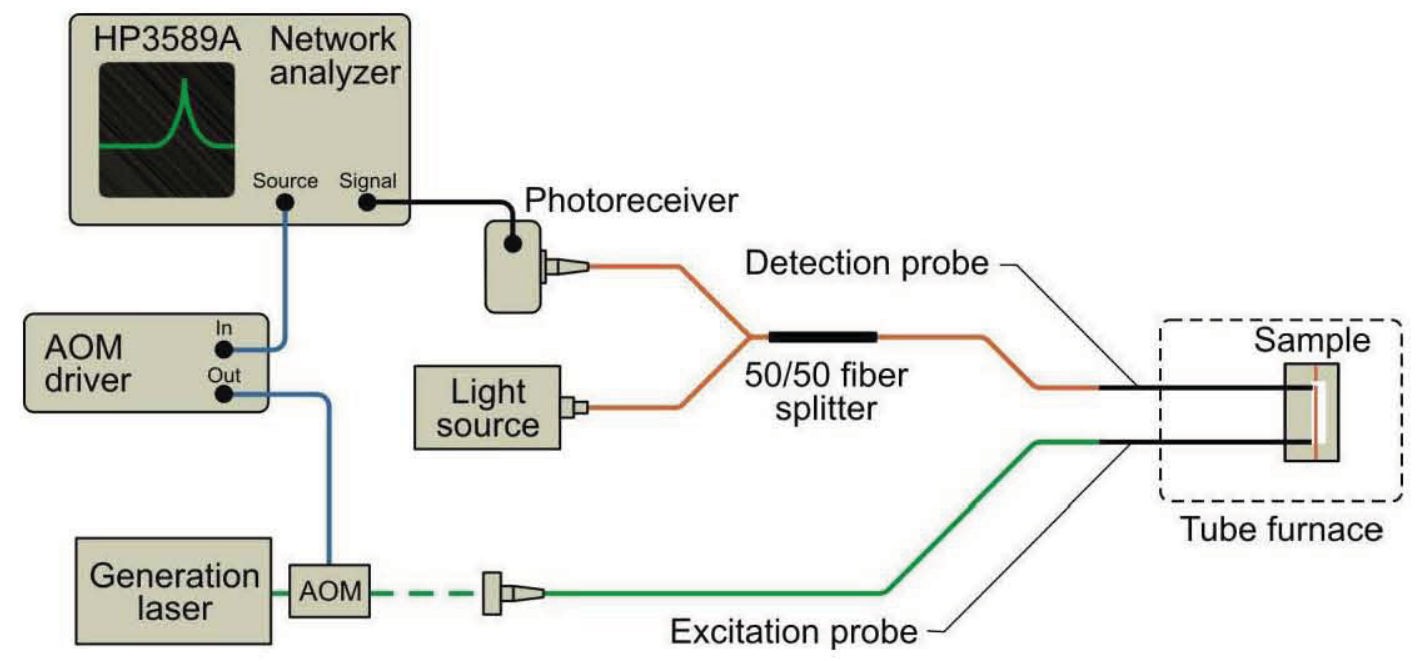

FIGURE 3. Experiment configuration.

bonded inside 1/16" diameter stainless steel tubes using a high temperature adhesive.

The probe tips where then inserted into the cap of the sample capsule and clamped in place. The sample capsule containing the cantilever beam along with both fiber optic probes was then inserted into a tube furnace. The temperature was controlled by manually adjusting the power to the furnace. Two K-type thermocouples were used to monitor the temperature using an HP 34970A Data Acquisition Unit. A flow of nitrogen purge gas was used to minimize oxidation of the sample and holder.

With the exception of the manual control of the furnace, the experiment was computer controlled using MatLab software such that a frequency scan was run using the network analyzer and temperatures recorded every 5 minutes. Initial scans were recorded after which power to the tube furnace was turned on. Power to the furnace was applied such that the temperature reached $350{ }^{\circ} \mathrm{C}$ in approximately 2.5 hours after which the power was incrementally reduced to allow cooling. After a total of approximately 7 hours the data acquisition was halted and the sample was allowed to cool overnight before final room temperature measurements were made.

\section{RESULTS}

The material used for the sample in this study consisted of high purity $(5 \mathrm{~N})$ copper with a textured microstructure imparted by repeatedly rolling until a $97.5 \%$ reduction was achieved. A sample was machined from this rolled copper sheet with the cantilever beam cut perpendicular to the roll direction. The initial room temperature frequency scan found the first flexural resonant mode of the beam to be $1,135 \mathrm{~Hz}$. The second and third flexural modes were measured at $7,110 \mathrm{~Hz}$ and $19,703 \mathrm{~Hz}$ respectively. To maintain a high frequency resolution, only the first flexural mode was monitored during the annealing process. Figure 4 shows the resonant frequency vs temperature for the duration of the experiment. Note that the resonant frequency initially decreases linearly with temperature as would be expected, but at approximately $150^{\circ} \mathrm{C}$, a sharp decrease in the resonant frequency occurs. The sharp decrease begins to slow at $200^{\circ} \mathrm{C}$ and by $250^{\circ} \mathrm{C}$ the resonant frequency again decreases slowly as the temperature increases. Upon cooling the resonant frequency increases near linearly back to $878 \mathrm{~Hz}$ at room temperature. Figure 5 shows room temperature scans of the first flexural mode of the cantilever beam both before and after the annealing process. The frequencies of the second and third modes after annealing were respectively $5,483 \mathrm{~Hz}$ and $15,305 \mathrm{~Hz}$. The decrease in the resonant frequency of the 


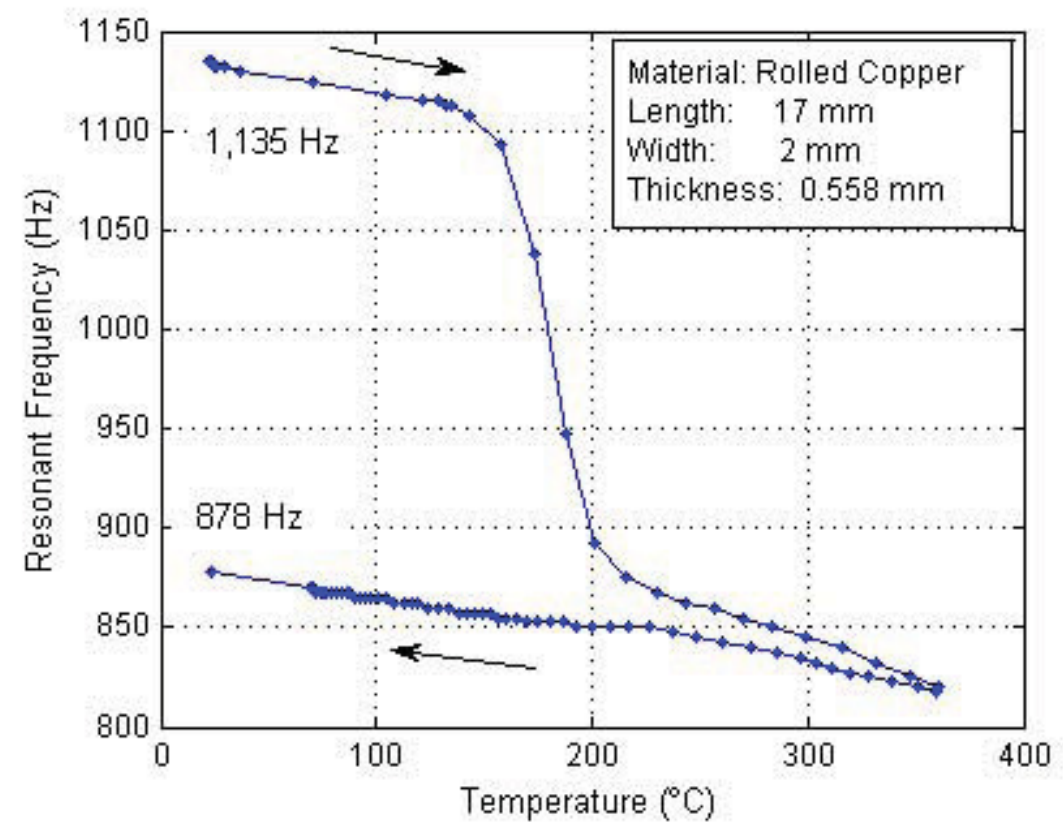

FIGURE 4. A plot of measured beam resonant frequency vs temperature

beam results from the recrystallization of the textured copper. Recrystallization is a process in which highly deformed grains in a material are replaced by new un-deformed grains which nucleate and grow consuming the original highly stressed, deformed grains. Recrystallization can, as in this case, lead to a significant change in elastic properties due to the altered material microstructure. Figure 6 shows electron backscatter diffraction (EBSD) micrographs of the sample material before and after annealing where shade is used to indicate grain orientation. Note the highly textured microstructure after rolling and the well defined cube texture after annealing [16].

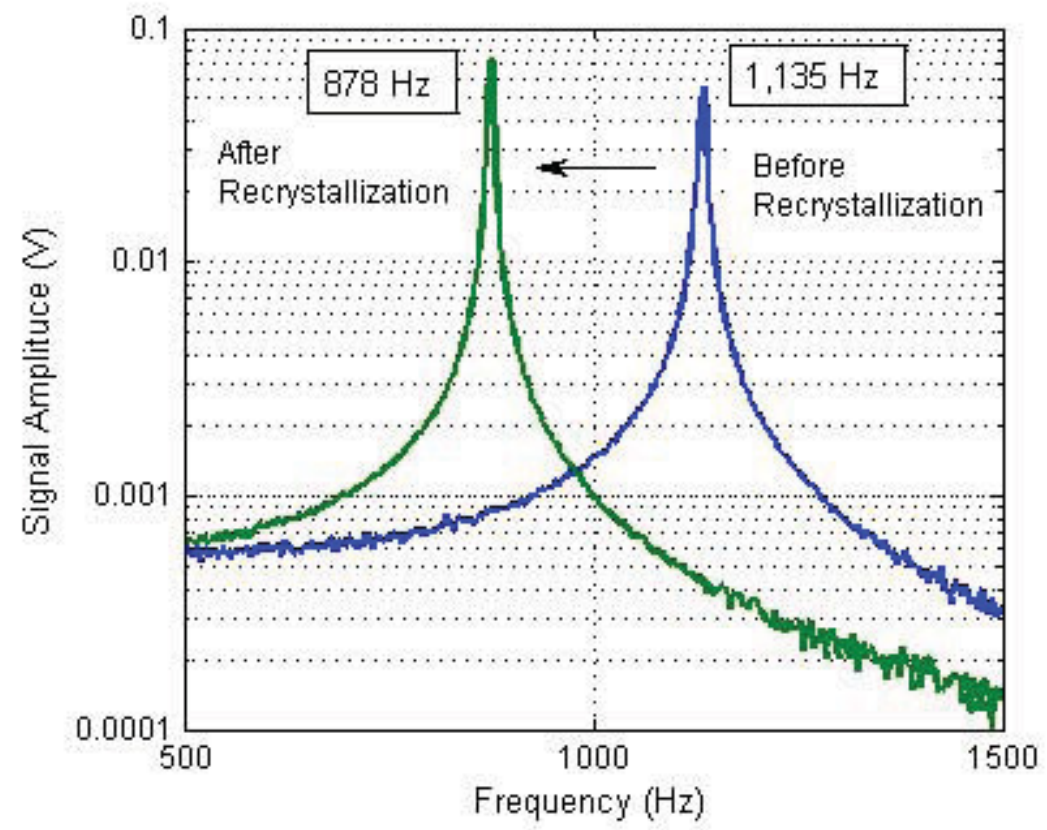

FIGURE 5. The shift in the resonant frequency before and after the annealing and recrystallizatoin of the copper sample 


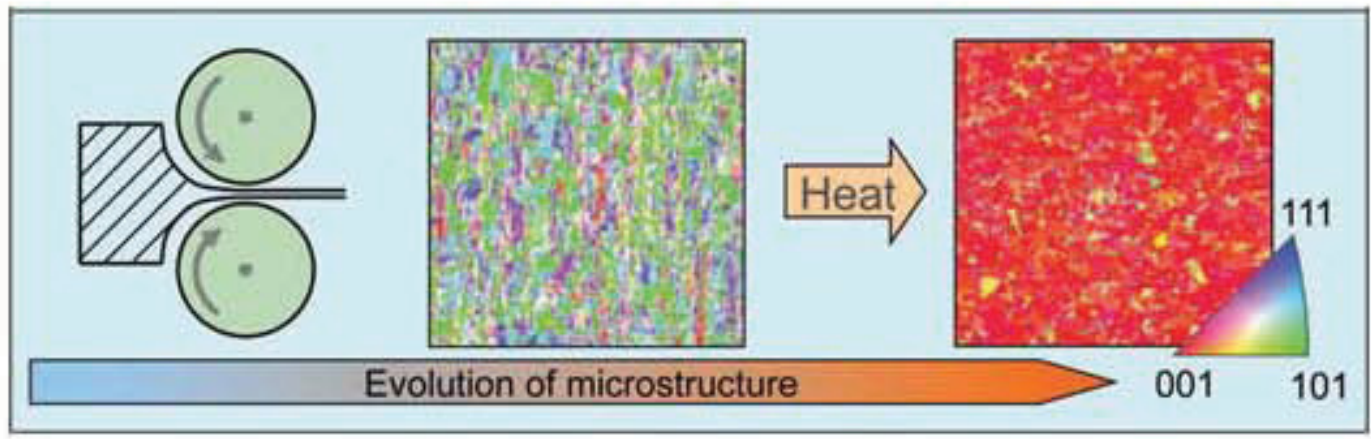

FIGURE 6. The microstructure evolution of a highly textured copper sample due to recrystalliztion during annealing [16].

The modulus of elasticity of the copper sample was calculated from equation (1) using the measured resonant frequencies both before and after annealing. The measured dimensions of the bearn used in the formula were: thickness, $t-0.558 \mathrm{~mm}$ and length, $\mathrm{L}^{-}$ $17.0 \mathrm{~mm}$ and a nominal density for copper of $\rho-8,940 \mathrm{~kg} / \mathrm{m}^{3}$ was used. The results are presented in Table 1 . In the rolled condition, the calculated modulus of $118.4 \mathrm{GPa}$ is consistent with values for polycrystalline copper found in the literature $(\sim 120 \mathrm{GPa})$, but less than the value determined using EBSD for this material $[16,17]$. The difference may be due to slight variations in the beam thickness or texture inherent in the highly deformed copper, or possibly due to beam damping or boundary conditions. There is a $2 \%$ variation in the values calculated using the first and third modes. It is believed this discrepancy is likely due to increasing inaccuracies in the Bernoulli-Euler model for higher order modes. In the annealed condition after recrystallization, the calculation yields a modulus of elasticity of $70.8 \mathrm{GPa}$. The modulus of elasticity for pure single crystal copper along a principle crystal axis is $67.1 \mathrm{GPa}$ [18]. Since the recrystallization process yields a highly oriented grain structure, but imperfect crystal, these results are certainly reasonable.

TABLE 1. Elastic modulus calculated from the measured resonant frequencies of the cantilever beam both before and after recrystallization.

\begin{tabular}{|c|c|c|c|c|c|}
\hline \multicolumn{2}{|c|}{} & \multicolumn{2}{c|}{ Sample in Rolled Condition } & \multicolumn{2}{c|}{ Sample in Annealed Condition } \\
\hline Mode & $\beta_{n} l$ & $\begin{array}{c}\text { Resonant } \\
\text { Frequency } \\
(\mathrm{Hz})\end{array}$ & $\begin{array}{c}\text { Calculated } \\
\text { Young's } \\
\text { Modulus } \\
(\mathrm{GPa})\end{array}$ & $\begin{array}{c}\text { Resonant } \\
\text { Frequency } \\
(\mathrm{Hz})\end{array}$ & $\begin{array}{c}\text { Calculated } \\
\text { Young's } \\
\text { Modulus } \\
(\mathrm{GPa})\end{array}$ \\
\hline 1 & 1.8751 & 1,135 & 118.4 & 878 & 70.8 \\
\hline 2 & 4.6941 & 7,110 & 118.3 & 5,483 & 70.3 \\
\hline 3 & 7.8548 & 19,703 & 115.9 & 15,305 & 69.9 \\
\hline
\end{tabular}




\section{SUMMARY}

We have presented a technique for the elastic properties determination of materials which could be used for real time in-reactor measurements. The technique involves the excitation of resonant flexural vibrations in a small cantilever beam using a chopped laser source. Detection is accomplished using a simple fiber optic lever. The technique was demonstrated by measuring the change in elastic modulus of highly textured copper during recrystallization at high temperature.

\section{ACKNOWLEDGEMENTS}

This work was sponsored by the U. S. Department of Energy through the Idaho National Laboratory (INL) - Laboratory Directed Research and Development Program under DOE Idaho Operations Office Contract DE-AC07-05ID14517.

\section{REFERENCES}

1. G. S. Was, Fundamentals of Radiation Materials Science, Springer, Berlin, 2007.

2. D. O. Thompson and D. K. Homes, J. Appl. Phys. 27, 713-723 (1956).

3. J. Rempe, H. MacLean, R. Schley et al., New In-Pile Instrumentation to Support Fuel Cycle Research and Development, INL/EXT-10-19149, Idaho National Laboratory (Jan 2011).

4. B. G. Kim, J. L. Rempe, J. F. Villard, S. Solstad, Nuclear Technology, 176, 155-187, (2011).

5. C. G. Lee and S. Okuda, Phys. Status Solidi A 164, 659 (1997).

6. ASTM E1875-08, ASTM International, West Conshohocken, PA, 2008.

7. ASTM C747-93, ASTM International, West Conshohocken, PA, 1993.

8. ASTM C623-92, ASTM International, West Conshohocken, PA, 1992.

9. H.E. Rosinger and I.G. Ritchie, J. of Testing and Evaluation, 2(3),131-138, (1974).

10. S. P. Timoshenko, History of Strength of Materials, Dover, New York, 1953.

11. J. W. Strutt, Theory of Sound, Macmillan, London, 1877.

12. S.M. Han, H. Benaroya, T. Wei, J. of Sound and Vibration, 225(5), 935-988, (1999).

13. K. F .Graff, Wave Motion in Elastic Solids, Dover, New York, 1991, p. 157.

14. I. G. Ritchie, J. of Sound and Vibration, 31(4), 453-468, (1973).

15. R. O. Cook, C. W. Hamm, Applied Optics, 18( 19), 3230-3241, (1979).

16. D.H. Hurley, et al. JAP 107, 063510 (2010)

17. D.H. Hurley, et al. JAP 111, 053527 (2012)

18. J. F. Nye, Physical Properties of Crystals - Their Representation by Tensors and Matrices, Oxford University Press, New York (1985), p. 144-148. 